\title{
Comment on "Modeling the electrical conduction in DNA nanowires: Charge transfer and lattice fluctuation theories"
}

\author{
M. Panahi ${ }^{1, *}$ and M. Chitsazanmoghaddam ${ }^{2}$ \\ ${ }^{1}$ Soft Matter Lab, Physics Department, Bilkent University, Cankaya, 06800 Ankara, Turkey \\ ${ }^{2}$ Department of Physics, Alzahra University, Tehran 19938, Iran
}

(Received 28 April 2015; published 1 April 2016)

\begin{abstract}
In a recent paper [S. Behnia and S. Fathizadeh, Phys. Rev. E 91, 022719 (2015)] an analytical approach is proposed for the investigation of the conductivity properties of DNA. The authors use mean Lyapunov exponent methods as the backbone of their approach and try to interpret properties of the system based on its behavior. Their interpretation regarding the change in nature of the mean Lyapunov exponent at the denaturation temperatures and discussions of stability and instability based on the mean Lyapunov exponent method are questioned. Moreover there is misunderstanding between mean Lyapunov exponent and Lyapunov exponent.
\end{abstract}

DOI: 10.1103/PhysRevE.93.046401

The mean Lyapunov exponent (MLE) method was first introduced by Shibata in 1997 and then further extended by him [1-3]. MLE expresses clearly how disordered spatial patterns are. The spatial patterns are more disordered when the values of the mean Lyapunov exponent are larger. Conversely, the spatial patterns are more ordered when the values of the mean Lyapunov exponent are smaller. Previously this method has been applied to some DNA models and there has been some success in parameter selection rules for Peyrard-Bishop models $[4,5]$.

In the case of MLE behavior with respect to the temperature, Behnia and Fathizadeh [6] coupled the system to a thermal bath and determined MLE as a function of temperature; this is shown in Fig. 5 of the paper. As the temperature approaches $340 \mathrm{~K}$, MLE increases, implying that the DNA chains are becoming more disordered. However, above $340 \mathrm{~K}$ the MLE decreases as temperature increases. This is very odd as it appears to violate one of the fundamentals of thermodynamics, that entropy increases with temperature (Fig. 1) [7]. Note the discontinuities in the graph at phase transitions, corresponding to the entropy change of transition.

Principally when a DNA chain couples with a thermal bath its entropy increases with temperature [8] and consequently high entropy is achieved when the system goes to a more disordered state. As the system becomes more disordered the values of MLE must increase [1]; however, in Fig. 5 of the paper, the MLE decreases for temperatures above $340 \mathrm{~K}$.

In the case of the MLE interpretation, the MLE method introduced by Shibata has no interpretations relating to stability-to-instability transitions, or for the sensitivity to the initial conditions [1]; there are relevant interpretations for the Lyapunov exponents. However, the whole paper is full of these concepts referring to behavior of the MLE.

It seems that the authors have confused the mean Lyapunov exponent (MLE) with the Lyapunov exponent (LE) or maximum Lyapunov exponents, which sometimes take the "MLE" acronym.

The Lyapunov exponent is a quantity that is very sensitive to initial conditions and characterizes the rate of separation

\footnotetext{
*m.panahi@chmail.ir
}

of infinitesimally close trajectories. Its sign determines the chaotic and stable regions of the dynamical systems.

The mean Lyapunov exponent and Lyapunov exponent are different conceptually. The most basic difference is the fact that in the Lyapunov exponent the system iterates in time and then the evaluation of the neighborhood trajectories can reveal how the dynamics of the system is [9]; in contrast to the Lyapunov exponent there is no way for time evaluated trajectories to take a role in the mean Lyapunov exponent. In fact, according to Shibata's papers [1-3], the mean Lyapunov exponent is the case only for a fixed point in time and expanding it to the whole dynamics is extremely incorrect.

Although the authors have explained Lyapunov exponent properties in the paper, they have never used it in the paper. This misunderstanding between LE and MLE causes a large incoherency in the whole paper.

The last point which should be mentioned here is the poor theoretical background of the MLE. Shibata did not provide enough theoretical justification to make his definition reliable [1-3]. Extending the LE theoretical basis to MLE is still questionable, so to avoid scientific fallacy it seems that the authors of the paper have to be more cautious interpreting the results by means of MLE.

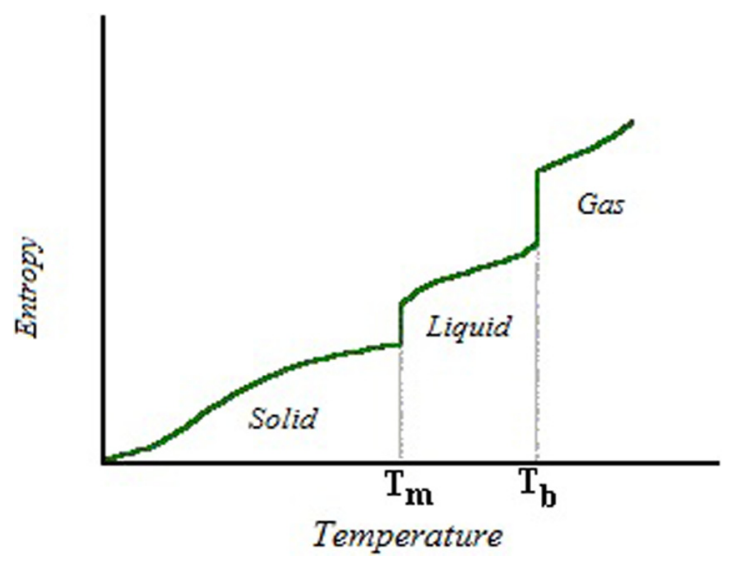

FIG. 1. The graph illustrates how the entropy of a substance increases with temperature [7]. 
Even though the authors have previously studied the DNA denaturation process successfully by means of multifractal analysis [10,11], using MLE for analyzing the denaturation process does not seem to have been done properly.
[1] H. Shibata, Physica A 264, 226 (1999).

[2] H. Shibata, Physica A 285, 325 (2000).

[3] H. Shibata, Physica A 292, 175 (2001).

[4] S. Behnia, M. Panahi, A. Mobaraki, and A. Akhshani, Phys. Lett. A 375, 1092 (2011).

[5] S. Behnia, M. Panahi, A. Akhshani, and A. Mobaraki, Phys. Lett. A 375, 3574 (2011).

[6] S. Behnia and S. Fathizadeh, Phys. Rev. E 91, 022719 (2015).
[7] T. Engel and P. Reid, Physical Chemistry, 3rd ed. (Pearson, New York, 2013).

[8] I. Rouzina and V. A. Bloomfield, Biophys. J. 77, 3242 (1999).

[9] E. Ott, Chaos in Dynamical Systems (University of Maryland, College Park, MD, 1993).

[10] S. Behnia, A. Akhshani, M. Panahi, A. Mobaraki, and M. Ghaderian, Phys. Rev. E 84, 031918 (2011).

[11] S. Behnia, A. Akhshani, M. Panahi, A. Mobaraki, and M. Ghaderian, Phys. Lett. A 376, 2538 (2012). 